\title{
Estrategias de aprendizaje, de la educación a la neurocognición
}

\section{Learning strategies, from education to neurocognition}

Recibido el 01 de agosto del 2016, aceptado el 02 de julio del 2017

*Martínez Flores, Fernanda Gabriela maga.mtzf@gmail.com ORCID: 0000-0002-2974-0876 *Delgado Sánchez, Ulises ulises.delgado@uaem.mx ORCID: 0000-0002-4318-0238 **Guerrero Benavides, Gabriela gabyguerrero@hotmail.com ****Hernández Padilla Eduardo eduhpad@gmail.com

*Centro de Investigación Transdiciplinar en Psicología, Universidad Autónoma del Estado de Morelos - México ***Benemérita Universidad Autónoma de Puebla - México

****Facultad de Comunicación Humana, UAEM - México
Para referenciar este artículo: Martínez, F. G., Delgado, U., Guerrero, G. y Hernández, E. (2017). Estrategias de aprendizaje, de la educacióna la neurocognición ConCiencia EPG, 2(2), 57-66.

\section{Resumen}

L as estrategias de aprendizaje es una de las líneas de investigación en diferentes niveles y contextos educativos, estas investigaciones se han desarrollado en todo el mundo, comenzando en un primer momento en la conceptualización y posteriormente en su evaluación. Estas estrategias consideran los procesos cognitivos como una parte fundamental de ellas, por lo que retoman a las neurociencias de una manera implícita, pues son inminente los procesos que van desde la motivación, el análisis y síntesis de la información hasta la planeación y control de la actividad, involucrando el funcionamiento de áreas corticales y subcorticales. El reto es el estudio de las estrategias de aprendizaje en poblaciones vulnerables que salen de los parámetros de la norma, presentando una organización dinámica de las funciones psicológicas superiores diferentes.

Palabras clave: estilos de aprendizaje, modelos, conceptualización, cognición

\section{Summary}

he learning strategies is one of the lines of research at different levels and educational contexts, these researches have been developed around the world, beginning at first in the conceptualization and later in their evaluation. These strategies consider the cognitive processes as a fundamental part of them, so they retake the neurosciences in an implicit way, since imminent processes ranging from motivation, analysis and synthesis of information to the planning and control of the activity, involving 
the functioning of cortical and subcortical areas. The challenge is the study of learning strategies in vulnerable populations that depart from the parameters of the norm, presenting a dynamic organization de different higher psychological functions.

Keywords: learning styles, models, conceptualization, cognition

\section{Introducción}

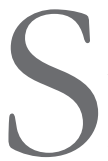

on diversas las investigaciones que se han realizado en relación a las estrategias de aprendizaje, abarcando diferentes niveles educativos como es secundaria, preparatoria y universidad. En los estudios de éste se han utilizado diversos instrumentos y se han vinculado con múltiples variables como es el rendimiento académico, el sexo, la autorregulación, perfil curricular y tipo de carrera, entre otros (Cepeda \& López, 2012; Delgado, Cárdenas, Flores \& Guzmán, 2014; Delgado \& Martínez, 2016, Delgado, Martínez \& Flores, 2016; Gargallo, 2002).

Las estrategias han sido importantes desde finales del siglo pasado y comienzos de este nuevo milenio, presentando diferencias en su conceptualización, así como en su clasificación, existiendo diversos modelos y propuestas de evaluación de las mismas que aportan información sobre el comportamiento de los estudiantes en relación a las tareas de aprendizaje.

\section{Conceptualización de las estrategias de aprendizaje}

El termino estrategias de aprendizaje ha sido en las últimas décadas muy usado en las áreas de psicología, educación e investigación. Gargallo, Almerich, Suárez y García (2012) toman la postura de afirmar que las estrategias de aprendizaje son un constructo multidimensional del que se han dado múltiples definiciones.

Hartley (1998) menciona que los estilos de aprendizaje es el camino en el que el individuo se acerca a diferentes tareas de aprendizaje, en tanto, las estrategias de aprendizaje son las estrategias que los estudiantes adoptan cuando estudian por lo que diferentes estrategias pueden ser seleccionadas por los estudiantes para la solución de diferentes tareas, mientras que los estilos son más automáticos (Cassidy, 2004). Por su parte, Gargallo, Almerich, Suarez y García (2012), las definen como un conjunto organizado, consciente e intencional de lo que hace el aprendiz para lograr con eficacia un objetivo de aprendizaje, en un contexto social dado, integrando elementos afectivo-motivacionales y de apoyo, metacognitivos y cognitivos.

En esta sintonía, Gázquez, Pérez, Ruiz, Miras y Florencio (2006), las consideran dentro del procesamiento de la información, viéndolas como un procedimiento o actividades mentales que van a facilitar el proceso de adquisición, codificación y recuperación de la información, existiendo, por lo tanto, estrategias cognitivas y metacognitivas. Las estrategias cognitivas son modalidades de trabajo intelectual que permite adquirir, codificar y recuperar la información, mientras que las estrategias metacognitivas son mediaciones del proceso cognitivo que permiten hacer consiente y autorregular dicho procesamiento, tomando decisiones más efectivas y logrando un aprendizaje en profundidad (Correa, Castro \& Lira, 2004).

Por otra parte, Genovard expresa que el término "estrategia" no es un concepto que naciera en la psicología, sino que ésta lo retomó de los términos usados en la milicia, que indicaba las actividades necesarias para llevar a cabo un plan previo de operaciones bélicas a gran escala, y el dominio de los diferentes estadios que esto suponía era denominado "táctica"; vinculándose implícitamente en esta conceptualización los términos de táctica y destreza (1990, en Valle, Barca, González \& Núñez,1999). Por ello el objetivo del presente artículo es hacer una recopilación de las principales propuestas que se han implementado para el estudio de las estrategias de aprendizaje.

Principales propuestas en la categorización de las estrategias de aprendizaje 
No solo han existido diferencias en la conceptualización de las estrategias de aprendizaje, de igual manera se han planteado modelos y evaluaciones que categorizan los tipos de estrategias de aprendizaje en las que los estudiantes se encuentran o hacen uso de ellas.

\section{a) Propuesta de J.B. Biggs, modelo $3 P$}

Un modelo clásico de los estilos de aprendizaje es el modelo 3P de Biggs (ver figura 1), es un modelo que surgió en los años 70s planteado por J.B Biggs quien colaboro en instituciones de Australia, Londres y Hong Kong, donde desarrollo su modelo de enseñanza (Biggs, Kember \& Leung, 2001; Rosário, Núñez, González, Almeira, Soares \& Rubio, 2005). Este modelo considera la vinculación entre la enseñanza y el aprendizaje, retomando principios básicos de Dunkin y Bidle (1974) (como se citó en Rosario et al, 2005) los cuales describen el proceso de aprendizaje y funcionamiento de un aula en tres fases: presagio, proceso y producto; de ahí el nombre del modelo de Biggs de "3P". Este modelo consta de cuatro componentes que se engloban en las tres fases.

1.-Factor de presagio: está formado por dos componentes; 1) características individuales de los alumnos, los cuales llegan con algunas competencias cognitivas, conocimientos, experiencias y motivaciones, 2) contexto de enseñanza, que se vincula con la institución, la metodología de ésta, la evaluación y experiencia del docente.

2.- Factor de proceso: la tarea de aprendizaje se maneja de forma diferente por el estudiante, de acuerdo a cómo éste interprete el contexto de enseñanza, a la luz de sus propias ideas y motivaciones, y la naturaleza de la tarea en cuestión. Tiene relación con el desarrollo de una actividad meta-cognitiva en la medida en que el estudiante utiliza la memoria y los procesos cognitivos superiores.

\begin{abstract}
3.- Factor de producto: corresponden a los resultados escolares, que pueden ser descritos cuantitativa (cuanto se aprende) o cualitativamente (lo bien que se aprende) (Barca, Peralbo \& Brenlla, 2004; Biggs, 1991; López \& Falchetti, 2009; Rosario et al, 2005)
\end{abstract}

Por ello, Biggs (1991) refiere que, para poder realizar las tareas, los estudiantes idean estrategias para resolver los problemas definidos por sus motivos. Esta combinación de motivos y estrategia se llama un "enfoque", siendo tres los planteados por éste modelo, los cuales son: el superficial, el profundo y el de logro (ver Figura 1) (Biggs, 1991; López \& Falchetti, 2009).

- Superficial: Este tipo de estudiantes se basa en la motivación extrínseca, se centra en los aspectos concretos y literales de los componentes de la tarea, y no en su significado o su relación con otras tareas previas.

- Profundo: los estudiantes se implican en un máximo entendimiento para satisfacer a la curiosidad, se centran en el significado que subyace a lo literal, buscando integrar las lecturas y discutir con los otros del tema para poder formular hipótesis de cómo se relaciona con otros aspectos.

- Logro: los estudiantes de logro se identifican a partir de altas calificaciones, se basan en la consecución de la mejora y presentan una organización de su tiempo, espacio y programan el trabajo que tienen que realizar de la manera más conveniente, aplicando "técnicas de estudio" (Biggs, 1991).

Específicamente para poder evaluar las variables del factor de proceso, J. Biggs creo los instrumentos de Learning Process Questionnaire (LPQ) y el Study Process Questionnaire (SPQ) (Biggs, 1991), realizando un replanteamiento de este último en 2001 (Biggs, Kember \& Leung, 2001). 


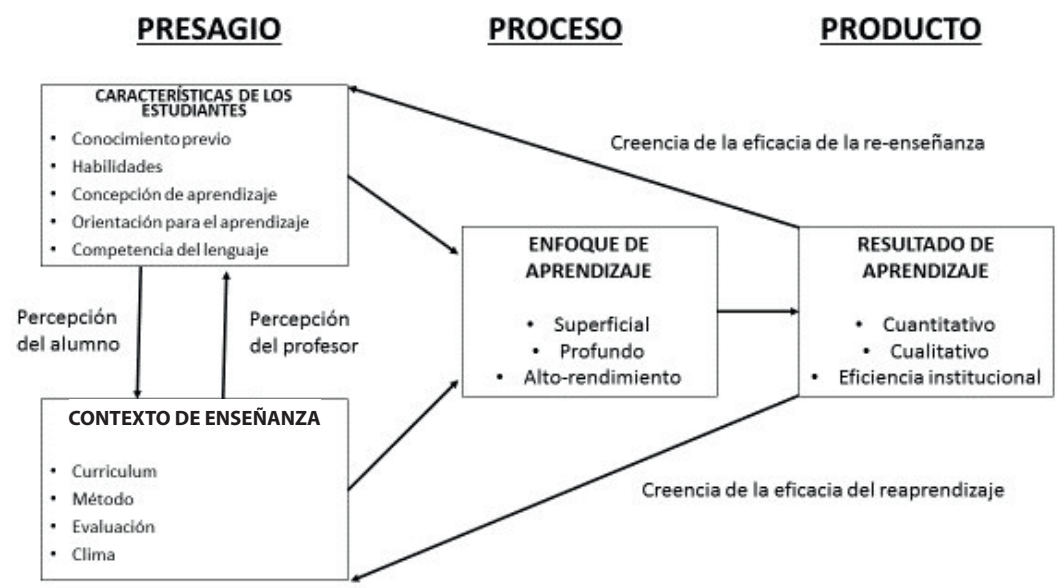

Figura 1. Modelo 3P de J.B. Biggs (Biggs, 1991)

\section{b) Propuesta de Valle, Barca, González y Núñez prueba CEPA}

Valle, Barca, González y Núñez (1999), retoman lo planteado por Genovard, concibiendo que las estrategias de aprendizaje suponen una secuencia de pasos o etapas a la hora de ponerlas en práctica, lo cual requiere que los individuos que las realizan dispongan de un conjunto de herramientas cognitivas que habitualmente se denominan "destrezas" es decir, aquellas capacidades que pueden concretarse en un comportamiento, puesto que se han ido desarrollando mediante un entrenamiento y luego en una práctica aplicada específica (Valle, Barca, González \& Núñez, 1999). Este grupo de investigadores clasificaron a las estrategias de aprendizaje en tres dimensiones: estrategias cognitivas, estrategias meta-cognitivas y estrategias de manejo de recursos.

- Estrategias cognitivas: Son las que integran el material nuevo con el conocimiento previo, siendo un conjunto de estrategias que se utilizan para aprender, codificar, comprender y recordar la información al servicio de una meta especifica de aprendizaje. En estas estrategias están la repetición, elaboración y organización de la información.

- Estrategias meta-cognitivas: Estas estrategias hacen referencia a la planificación, control y evaluación por parte de los estudiantes de su propia cognición.

- Estrategias de manejo de recursos: las cuales son estrategias de apoyo que incluyen diferentes tipos de recursos que contribuyen a que la resolución de la tarea se lleve a buen término (Valle, González, Barca \& Núñez, 1996).

Crearon la escala del Sistema Integrado de Evolución de Atribuciones Causales y Procesos de Aprendizaje (SIACEPA), en la que se integra la Subescala de Evaluación de Atribuciones Causales Multidimensionales (EACM) y la Subescala de Procesos y Estrategias de Aprendizaje/Enfoques de Aprendizaje (CEPA), ésta última escala relaciona con las estrategias de aprendizaje constan de 6 factores que retoma el modelo 3P de J. Biggs, con un total de 35 ítems. Con este instrumento se clasifican a los 
estudiantes en seis tipos diferentes acordes con el tipo de estrategia que predomina en ellos.

1. Enfoque Profundo/Logro/Estrategias con Orientación al Significado: los estudiantes buscan una necesidad de rendimiento, con una intención de sobresalir logrando una rentabilidad a su trabajo, organizan y distribuyen su tiempo para tener mejores resultados en sus exámenes. Utilizan estrategias de logro (interés en completar apuntes y búsqueda de la compresión de los mismos, etc...) y estrategias profundas (interés hacia los temas nuevos, preocupación por relacionar los contenidos, transferencia a la vida real).

2. Enfoque Motivacional Mixto-1. Orientación al Significado: en estos estudiantes domina la intención de comprender, sobresalir y tener un buen rendimiento. Presentan una motivación intrínseca para comprender y relacionar los diferentes contenidos, se dejan llevar por las circunstancias por lo que el resultado no es siempre el esperado.

3. Enfoque Motivacional Mixto-2. Orientación al Significado: los estudiantes se realizan macro-guías de aprendizaje que ordenan y estructuran las tareas de estudio, presentan un buen rendimiento académico y poseen una motivación extrínseca por lo que se adaptan a las circunstancias en su proceso de aprendizaje.

4. Enfoque Profundo Puro/Orientación al Significado: los estudiantes presentan una motivación intrínseca, leen a profundidad y amplían sus lecturas, por lo que interactúan con los contenidos relacionando los nuevos conocimientos con las experiencias e ideas previas, tratan de examinar y fundamentar la lógica de los argumentos.

5. Enfoque Superficial/Estrategias con Orientación Superficial: Los estudiantes se centran en la naturaleza reproductiva de los contenidos de aprendizaje, omiten o evitan las interrelaciones que pueden existir entre los componentes de las tareas o contenidos, se limitan solo a cumplir con los mínimos requisitos exigidos, presentando una motivación extrínseca, por lo que tienden a la reproducción mecánica de aprendizaje memorizando temas/ hechos/ procedimientos, sólo para pasar las pruebas o exámenes.

6. Enfoque Motivacional de Logro/ Orientación Superficial: estos estudiantes consideran importante obtener altas calificaciones y compiten para logarlas, para ellos es importante satisfacer los requisitos formales de la presentación de trabajos y la puntualidad en entrega; pero estos estudiantes no poseen estrategias adecuadas para conseguir estos objetivos, limitándose a lo esencial y a la reproducción, presentando un aprendizaje memorístico/mecánico. (Abalde, Barca, Muñoz \& Ziemer, 2009; Barca, Pesutti, Brenilla \& Santamaría, 2000; Barca, Peralbo. Brenlla, 2004).

Esta escala ha sido aplicada en países de lengua romance como España y Brasil (Abalde, Barca, Muñoz \& Ziemer, 2009).

\section{c) Propuesta de Gargallo, Suárez y Ferraras prueba CEVEAPEU}

Gargallo, Suárez y Ferraras (2007) crearon el Cuestionario de Evaluación de las Estrategias de Aprendizaje de los Estudiantes Universitarios (CEVEAPEU) el cual comenzó con 165 ítems, después se redujo a 94 para terminar con un total de 88 ítems con respecto a los cuales el sujeto se pronuncia en una escala Likert. El cuestionario está organizado en dos escalas, seis subescalas y 25 estrategias (Bozo \& De la O, 2012).

A continuación, se mencionará como están conformadas cada una de estas escalas. 


\section{Escala de las estrategias afectivas, de apoyo y control (automanejo)}

Esta escala tiene cuatro subescalas.

- Estrategias motivacionales: incluye la motivación intrínseca, extrínseca, el valor de la tarea, atribuciones internas, atribuciones externas, autoeficacia y expectativas, concepción de la inteligencia.

- Componentes afectivos: consta del estado físico y anímico positivo, así como del control de la ansiedad.

- Estrategias metacognitivas: está formada por los conocimientos de objetivos y criterios de evaluación, la planeación, autoevaluación, el control y la autorregulación.

- Estrategias del control del contexto, interacción social y manejo de recursos: control del contexto, habilidades de interacción social y aprendizaje con compañeros.

\section{Escala de las estrategias relacionadas con el procesamiento de la información Está integrada por dos subescalas.}

- Estrategia de búsqueda y selección de la información: implica el conocimiento de fuentes y búsqueda de información, así como la selección de información.

- Estrategias de procesamiento y uso de la información: consta de los aspectos de adquisición de la información, elaboración, organización, personalización y creatividad, pensamiento crítico, almacenamiento memorístico, almacenamiento simple repetición, transferencia y el manejo de recursos para usar la información adquirida (Gargallo et al, 2012; Gargallo et al, 2007; Gargallo, Suárez y Pérez, 2009). d) Propuesta de Weinstein, Zimmerman y Palmer prueba LASSI

En países de habla inglesa también se han realizado investigaciones con respecto a las estrategias de aprendizaje partiendo del modelo de Weinstein (Badenier, 2003), el cual se ubica en el marco del modelo del procesamiento de la información, donde la codificación de la información en el proceso de la enseñanza puede ser analizada dentro de cuatro componentes principales:

- Selección: el estudiante pone atención a una información y transfiere esta información a la memoria de trabajo.

- Adquisición: el estudiante transfiere la información de la memoria de trabajo a la memoria a largo plazo para un almacenamiento permanente.

- Construcción: en donde el alumno construye activamente conexiones entre ideas, esta construcción implica el desarrollo de un esquema u organización resumida y coherente que mantiene la información.

- Integración: el estudiante busca en la memoria a largo plazo el conocimiento previo y transfiere este conocimiento a la memoria de trabajo; de esta manera puede construir conexiones entre la información que ingresa y el conocimiento previo (Weinstein \& Palmer, 1990).

Con ello surgió el Learning and Study Strategies Inventory (LASSI), tanto para estudiantes de educación secundaria, como de universidad, este cuestionario fue propuesto por Weinstein, Zimmerman y Palmer en 1987; consta de 77 ítems y 10 escalas que son: actitud, motivación, gestión del tiempo, ansiedad, concentración, procesamiento de la información, selección de ideas principales, ayudas de estudio, autoevaluación, preparación y presentación de pruebas (Beltrán, 2003; Weinstein \& Palmer, 1990). 


\section{Artículos Teóricos}

e) Propuesta de Sánchez y Gallego prueba ACRA

Ésta postura se centra en los procesos mentales que el alumno pone en macha a la hora de estudiar y que tiene en cuenta las últimas aportaciones de la psicología de la inteligencia, de la memoria y psicología del aprendizaje (Del Caño, Román \& Foces, 2000),

Este enfoque lo comenzaron a trabajar en España José María Sánchez y Sagrario Gallego Rico, del departamento de Psicología de la Universidad de Valladolid, mediante la creación del instrumento llamado ACRA que se relaciona con los procesos de adquisición, codificación, recuperación y apoyo al procesamiento (Beltrán, 2003).

El cuestionario ACRA está constituido por cuatro dimensiones independientes que evalúan el uso que habitualmente hacen los estudiantes: 1) siete estrategias de adquisición de la información; 2) trece estrategias de codificación de la información; 3) cuatro estrategias de recuperación y 4) nueve estrategias de apoyo al procesamiento, estos se observan en la Tabla 1 (Albo, 2012; Correa, Castro \& Lira, 2004).

Tabla 1

Escalas del instrumento ACRA

\begin{tabular}{|c|c|c|c|}
\hline \multicolumn{3}{|c|}{ Evaluación de estrategias de carácter cognitivo } & \multirow{2}{*}{$\begin{array}{l}\text { Es de naturaleza } \\
\text { cognitiva y no cognitiva } \\
\text { Escala IV: Estrategias de } \\
\text { apoyo al procesamiento } \\
\text { de información }\end{array}$} \\
\hline $\begin{array}{l}\text { Escala I: Estrategias } \\
\text { de adquisición de } \\
\text { información. }\end{array}$ & $\begin{array}{l}\text { Escala II: Estrategias } \\
\text { de codificación de } \\
\text { información }\end{array}$ & $\begin{array}{l}\text { Escala III: Estrategias } \\
\text { de recuperación de } \\
\text { información }\end{array}$ & \\
\hline $\begin{array}{l}\text { 1. Exploración. } \\
\text { 2. Subrayado lineal. } \\
\text { 3. Subrayado } \\
\text { ideosincrásico. } \\
\text { 4. Epigrafiado. } \\
\text { 5. Repaso en voz alta. } \\
\text { 6. Repaso mental. } \\
\text { 7. Repaso reiterado. }\end{array}$ & $\begin{array}{l}\text { 8. Nemotecnias. } \\
\text { 9. Relaciones intra } \\
\text { contenido. } \\
\text { 10. Relaciones } \\
\text { compartidas. } \\
\text { 11. Imágenes. } \\
\text { 12. Metáforas. } \\
\text { 13. Aplicaciones. } \\
\text { 14. Auto preguntas. } \\
\text { 15. Paráfrasis. } \\
\text { 16. Agrupamientos. } \\
\text { 17. Secuencias. } \\
\text { 18. Mapas conceptuales. } \\
\text { 19. Diagramas. }\end{array}$ & $\begin{array}{l}\text { 20. Búsqueda de } \\
\text { codificaciones. } \\
\text { 21. Búsqueda de } \\
\text { indicios. } \\
\text { 22. Planificación de } \\
\text { respuestas. } \\
\text { 23. Respuesta escrita. } \\
\text { 31. Motivación } \\
\text { intrínseca y extrínseca. } \\
\text { 32. Motivación de } \\
\text { escape. }\end{array}$ & $\begin{array}{l}\text { 24. Autoconocimiento. } \\
\text { 25. Automanejo / } \\
\text { Planificación. } \\
\text { 26. Automanejo/ } \\
\text { Regulación y evaluación. } \\
\text { 27. Autoinstrucciones. } \\
\text { 28. Autocontrol. } \\
\text { 29. Contradistractoras. } \\
\text { 30. Interacciones sociales }\end{array}$ \\
\hline
\end{tabular}

(Correa, Castro \& Lira, 2004). 


\section{Artículos Teóricos}

En la Tabla 2 se puede observar algunos modelos planteados de las estrategias de aprendizaje y su relación entre ellos, esto a partir de un análisis realizado de los diversos modelos.

Tabla 2

Taxonomía de los modelos de las estrategias de aprendizaje

\begin{tabular}{|c|c|c|c|c|c|c|c|}
\hline \multirow{3}{*}{ MODELO } & \multicolumn{3}{|c|}{$\begin{array}{c}\text { Valle, Barca, González \& Núñez } 1999 \\
\text { CEPA }\end{array}$} & \multicolumn{4}{|c|}{$\begin{array}{l}\text { Biggs, } 1970 \\
\text { Modelo 3P }\end{array}$} \\
\hline & \multirow{2}{*}{$\begin{array}{l}\text { Estrategias } \\
\text { cognitivas }\end{array}$} & \multirow{2}{*}{$\begin{array}{l}\text { Estrategias } \\
\text { metacognitivas }\end{array}$} & \multirow{2}{*}{$\begin{array}{l}\text { Estrategias } \\
\text { de manejo } \\
\text { de recursos }\end{array}$} & \multirow{2}{*}{$\begin{array}{c}\text { Factor } \\
\text { presagio }\end{array}$} & \multicolumn{2}{|c|}{ Factor proceso } & \multirow{3}{*}{$\begin{array}{l}\text { Factor } \\
\text { producto }\end{array}$} \\
\hline & & & & & Superficial & Profunda & \\
\hline CEPA & + & + & + & & + & + & \\
\hline CEVEAPEU & + & + & + & + & + & + & \\
\hline ACRA & + & + & + & & & & \\
\hline LASSI & + & + & + & & + & & \\
\hline
\end{tabular}

\section{Discusión y Conclusiones}

Aún y cuando las estrategias de aprendizaje se han planteado desde la línea educativa, en busca de indagar la manera en la que los estudiantes logran apropiarse del aprendizaje, es inminente que han puesto a relucir la importancia de los procesos psicológicos superiores, entre los que destacan, la memoria, el razonamiento, el análisis y síntesis de la información, la planeación, así como la importancia de la esfera efectivo emocional.

Desde la conceptualización y evaluación de las estrategias de aprendizaje se considera el funcionamiento dinámico de diversas áreas cerebrales, por ejemplo, la organización, planeación y control de la actividad, así como la metacognición que se desarrolla en los lóbulos frontales, siendo el tercer bloque funcional propuesto por Luria (1988), existiendo a su vez conducta volitiva en la intencionalidad que mencionan Almerich, Suarez y García (2012).

Gázquez, Pérez, Ruiz, Miras y Florencio (2006) mencionan el procesamiento de la información el cual es retomado en diversos modelos, este procesamiento, para llevarse a cabo necesita del almacén de memoria, tanto a corto plazo y largo plazo siendo necesarias las áreas primarias, secundarias y terciarias del segundo bloque funcional que propone Luria, así como estructuras hipocampales y los fascículos de conexión inter e intra hemisférica.

Por otra parte, como lo han mencionado autores como Azcoaga, Derman e Iglesias (1997) en los dispositivos básicos del aprendizaje, las estrategias de aprendizaje consideran elementos afectivo-emocionales los cuales se relacionan con el sistema límbico, por medio del primer bloque funcional de activación (Luria, 1988), involucrando no solo procesos de sueño, vigilia y homeostasias fisiológica, sino que también, la motivación como el generador de la actividad (Leontiev, 1975).

Si bien, queda claro el papel que juegan las áreas cerebrales en las estrategias de aprendizaje, estas se han estudiado en lo que se considera la "norma", todo aquel estudiantes que está dentro de los parámetros esperados en la funcionalidad, pero que pasa con los estudiantes que salen de esa norma como los de pueblos originarios que tienden a dominar dos idiomas y por lo cual sus funciones psicológicas se organizan de manera 
distinta; así mismo estudiantes sordos, ciegos, adultos mayores, o con actitudes sobresalientes pertenecientes a otro grupo minoritarios, esta es una de las limitaciones que los modelos de estrategias no han considerado, siendo una ventana de oportunidad a desarrollar en futuras investigaciones.

A manera de conclusión se puede decir que las estrategias han dado información sobre la manera

\section{Referencias}

Abalde, E., Barca, A., Muñoz, J. \& Ziemer, M. (2009). Rendimiento académico y enfoque de aprendizaje: una aproximación a la realidad de la enseñanza superior brasileña en la región norte. Revista de Investigación Educativa, 27(2), 303-319.

Albo, G. (2012). ACRA: escalas de estrategias de aprendizaje de los estudiantes del curso de producción animal I. Facultad de Ciencias Agrarias y Forestales (UNLP) IV Congreso Nacional y III Congreso Internacional de Enseñanza de 992 las Ciencias Agropecuarias, Argentina.

Azcoaga, J., Derman, B. \& Iglesias, P. (1997). Alteración del aprendizaje escolar, diagnóstico, fisiopatología y tratamientos. Buenos Aires: Paidós.

Badenier, C. (2003). Confiabilidad y Validez del Learning and Study Strategies Inventory (LASSI) en una muestra de estudiantes de la regio metropolitana en Chile. Psykhe, 12(2), 193-206.

Barca, A., Peralbo, A. \& Brenlla, J. (2004). Atribuciones causales y enfoques de aprendizaje: la escala SIACEPA. Psicothema, 16(1), 94-103. en la que los estudiantes adoptan herramientas para aprender $y$ ha pasado de lo cognitivo a un objetivo educativo, por ello, es importante que se desarrollen programas de entrenamiento en estrategias por parte de las Universidades en los programas de tutoría, programas que deben ser inclusivos para dar oportunidad a todos los estudiantes.

Barca, A., Pesutti, C., Brenilla, J. \& Santamaría, S. (2000). Propiedades psicométricas de la escala SIACEPA (Sistema Integrado de Evaluación de atribuciones causales y procesos de aprendizaje) en una muestra de alumnos de educación secundaria de Brasil. V Congreso Galego- Portugués de Psicopedagoxia actas (comunicación e Posters), 4(6).

Beltrán, J. (2003). Estrategias de aprendizaje. Revista de educación, 332, 55-73.

Biggs, J. (1991). Approaches to Learning in Secondary and Tertiary Students in Hong Kong: Some Comparative Studies. Educational Research Journal, 6, 27-39.

Biggs, J.B., Kember, D. \& Leung, D.Y. (2001). The Revised Two Factor Study Process Questionnaire: R-SPQ-2F. British Journal of Educational Psychology, 71, 133-149.

Bozo, A. \& De la O, M. (2012). Motivos, actitudes y estrategias de aprendizaje: aprendizaje motivado en alumnos universitarios. Revista de currículum y formación del profesorado, 16(1), 125-142.

Cassidy, S. (2004). Learning styles: Anoverview of theories, models, and measures. Educational Psychology, 24(4), 419-444. 
Cepeda, M. \& López, M. (2012). Evaluación de estrategias de aprendizaje y habilidades verbal en una muestra de estudiantes universitarios. Enseñanza Investigación en Psicología, 11(1), 117-135.

Correa, M., Castro, E. \& Lira, H. (2004). Estudio descriptivo de las estrategias cognitivas y metacognitivas de los alumnos y alumnas de primer año de pedagogía en enseñanza media de la universidad Bío-Bío. Theoria, 13, 103-110.

Del Caño, M., Román, J. \& Foces, J. (2000). Estrategias de aprendizaje de las matemáticas: enseñanza explicita Vs enseñanza implícita y estilos de solución de problemas. Revista de Psicodidáctica, 10, 47-58.

Gargallo, B. (2002). Estrategias de aprendizaje, rendimiento académico y otras variables relevantes en estudiantes universitarios. Revista de psicología general y aplicada, 59(1), 109-130.

Gargallo, B., Almerich, G., Suárez, J. \& García, E. (2012). Estrategias de aprendizaje en estudiantes universitarios excelentes y medios. Su evolución a lo largo del primer año de carrera. Revista electrónica de Investigación y evaluación Educativa, 18(2), 1-22.

Gargallo, B., Suárez, J. \& Ferreras, A. (2007). Estrategias de aprendizaje y rendimiento académico en estudiantes universitarios. Revista de Investigación Educativa, 25(2), 421-441.

Gargallo, B., Suárez, J. y Pérez, C. (2009). El cuestionario CEVEAPEU. Un instrumento para la evaluación de las estrategias de aprendizaje de los estudiantes universitarios. Revista electrónica de Investigación y evaluación Educativa. 15(2).

Gázquez, J., Pérez, M., Ruiz, M., Miras, F. \& Florencio, V. (2006). Estrategias de aprendizaje en estudiantes de enseña secundaria obligatoria y su relación con la autoestima. International Journal of Psychological Therapy, 6(1), 51-62.

Hartley, J. (1998). Learning and studying: a research perspective. London: Routledge.

Leontiev, A. (1975). Actividad, consciencia, personalidad. Moscú: Universidad Estatal de Moscú.

López, M. \& Falchetti, E. (2009). Estilos de aprendizaje. Relación con motivación y estrategias. Revista de estilos de aprendizaje, 4(4).

Luria, A. R. (1988). El cerebro en acción. Buenos Aires: Orbis

Rosário, P., Núñez, J., González, J., Almeira, L., Soares, S. \& Rubio, M. (2005). El aprendizaje escolar examinado desde la perspectiva del "Modelo 3P" de J. Biggs. Psicothema, 17(1), 20-30.

Valle, A., Barca, A., González, R. \& Núñez, J. (1999). Las estrategias de aprendizaje. Revisión teórica y conceptual. Revista latinoamericana de psicología, 31(3), 425-461.

Weinstein. C. \& Palmer. D. (1990). Learning and Study Strategies Investory- High School Version. User's Manual. H\&H Publishing Company, Inc. Florida 